Bull. Korean Math. Soc. 46 (2009), No. 5, pp. 895-903

DOI 10.4134/BKMS.2009.46.5.895

\title{
HYPERSURFACES OF ALMOST $r$-PARACONTACT RIEMANNIAN MANIFOLD ENDOWED WITH SEMI-SYMMETRIC METRIC CONNECTION
}

\author{
Jae-BoK Jun* And Mobin Ahmad
}

\begin{abstract}
We define a semi-symmetric metric connection in an almost $r$-paracontact Riemannian manifold and we consider invariant, non-invariant and anti-invariant hypersurfaces of an almost $r$-paracontact Riemannian manifold endowed with a semi-symmetric metric connection.
\end{abstract}

\section{Introduction}

Let $\nabla^{*}$ be a linear connection in an $n$-dimensional differentiable manifold $M$. The torsion tensor $T$ of $\nabla^{*}$ is given by

$$
T(X, Y)=\nabla_{X}^{*} Y-\nabla_{Y}^{*} X-[X, Y]
$$

for all vector fields $X$ and $Y$ in $M$ and is of type $(1,2)$. The connection $\nabla^{*}$ is symmetric if its torsion tensor $T$ vanishes, otherwise it is non-symmetric. The connection $\nabla^{*}$ is metric connection if there is a Riemannian metric $g$ in $M$ such that $\nabla^{*} g=0$, otherwise it is non-metric. It is well known that a linear connection is symmetric if it is the Levi-Civita connection.

In [7], [8], A. Friedmann and J. A. Schouten introduced the idea of a semisymmetric linear connection in a differentiable manifold. A linear connection is said to be semi-symmetric if its torsion tensor $T$ is of the form

$$
T(X, Y)=u(Y) X-u(X) Y,
$$

where $u$ is a 1 -forms. In [9], K. Yano considered a semi-symmetric metric connection and studied some of its properties. Almost $r$-paracontact structures were defined by A. Bucki and Miernowski in [5]. In [4], A. Bucki introduced $r$-paracontact structures of $P$-Sasakian type. Properties of hypersurface of almost $r$-paracontact Riemannian manifold were studied by A. Bucki in [3]. M. Ahmad, J.-B. Jun, and A. Haseeb studied some properties of hypersurfaces of almost $r$-paracontact Riemannian manifold endowed with a quarter symmetric

Received July 23, 2008; Revised November 7, 2008.

2000 Mathematics Subject Classification. 53D12, 53C05.

Key words and phrases. hypersurface, almost $r$-paracontact Riemannian manifold, semisymmetric metric connection.

* Partially supported by Kookmin University 2009. 
metric connection in [1]. Also M. Ahmad and C. Ozgur studied those of them endowed with a semi-symmetric non-metric connection in [2].

In this paper we study properties of hypersurfaces of almost $r$-paracontact Riemannian manifold endowed with a semi-symmetric metric connection.

The paper is organized as follows: In Section 2, we give a brief introduction about an almost $r$-paracontact Riemannian manifold. In Section 3, we show that the induced connection on a hypersurface of an almost $r$-paracontact Riemannian manifold with semi-symmetric metric connection with respect to the normal is also a semi-symmetric metric connection. We find the characteristic properties of invariant, non-invariant and anti-invariant hypersurfaces of almost $r$-paracontact Riemannian manifold endowed with a semi-symmetric metric connection.

\section{Preliminaries}

Let $M$ be an $n$-dimensional Riemannian manifold with a positive definite metric $g$. If on $M$ there exist a tensor field $\phi$ of type $(1,1), r$ vector fields $\xi_{1}, \xi_{2}, \ldots, \xi_{r}(n>r), r$-forms $\eta^{1} \eta^{2}, \ldots, \eta^{r}$ such that

$$
\begin{gathered}
\eta^{\alpha}\left(\xi_{\beta}\right)=\delta_{\beta}^{\alpha}, \quad \alpha, \beta \in(r)=\{1,2,3, \ldots, r\}, \\
\phi^{2}(X)=X-\eta^{\alpha}(X) \xi_{\alpha}, \\
\eta^{\alpha}(X)=g\left(X, \xi_{\alpha}\right), \quad \alpha \in(r), \\
g(\phi X, \phi Y)=g(X, Y)-\Sigma_{\alpha} \eta^{\alpha}(X) \eta^{\alpha}(Y),
\end{gathered}
$$

where $X$ and $Y$ are vector fields on $M$ and $a^{\alpha} b_{\alpha} \stackrel{\text { def }}{=} \Sigma_{\alpha} a^{\alpha} b_{\alpha}$, then the structure $\Sigma=\left(\phi, \xi_{\alpha}, \eta^{\alpha}, \mathrm{g}\right)_{\alpha \in(r)}$ is said to be an almost $r$-paracontact Riemannian structure on $M$ and $M$ is an almost r-paracontact Riemannian manifold [5]. From (2.1) through (2.4), we also have:

$$
\begin{gathered}
\phi\left(\xi_{\alpha}\right)=0, \quad \alpha \in(r), \\
\eta^{\alpha} \circ \phi=0, \quad \alpha \in(r), \\
\Phi(X, Y) \stackrel{\text { def }}{=} g(\phi X, Y)=g(X, \phi Y) .
\end{gathered}
$$

An almost $r$-paracontact Riemannian manifold $M$ equipped with the Riemannian connection $\nabla^{*}$ with a structure $\Sigma=\left(\phi, \xi_{\alpha}, \eta^{\alpha}, \mathrm{g}\right)_{\alpha \in(r)}$ is said to be of $S$-paracontact type if

$$
\Phi(X, Y)=\left(\nabla_{Y}^{*} \eta^{\alpha}\right)(X)
$$

for all $\alpha \in(r)$. An almost $r$-paracontact Riemannian manifold $M$ with a structure $\Sigma=\left(\phi, \xi_{\alpha}, \eta^{\alpha}, \mathrm{g}\right)_{\alpha \in(r)}$ is said to be of P-Sasakian type if it satisfies 
(2.6) and

$$
\begin{aligned}
\left(\nabla_{Z}^{*} \Phi\right)(X, Y)= & -\Sigma_{\alpha} \eta^{\alpha}(X)\left[g(Y, Z)-\Sigma_{\beta} \eta^{\beta}(Y) \eta^{\beta}(Z)\right] \\
& -\Sigma_{\alpha} \eta^{\alpha}(Y)\left[g(X, Z)-\Sigma_{\beta} \eta^{\beta}(X) \eta^{\beta}(Z)\right]
\end{aligned}
$$

for all vector fields $X, Y$ and $Z$ on $M$ [4]. The conditions (2.6) and (2.7) are equivalent respectively to

$$
\phi X=\nabla_{X}^{*} \xi_{\alpha}
$$

and

$$
\begin{aligned}
\left(\nabla_{Y}^{*} \phi\right)(X)= & -\Sigma_{\alpha} \eta^{\alpha}(X)\left[Y-\eta^{\alpha}(Y) \xi_{\alpha}\right] \\
& -\left[g(X, Y)-\Sigma_{\alpha} \eta^{\alpha}(X) \eta^{\alpha}(Y)\right] \Sigma_{\beta} \xi_{\beta}
\end{aligned}
$$

for all $\alpha \in(r)$. On the other hand, a semi-symmetric metric connection $\nabla$ on $M$ is defined as

$$
\nabla_{X} Y=\nabla_{X}^{*} Y+\eta^{\alpha}(Y) X-g(X, Y) \xi_{\alpha}
$$

for any $\alpha \in(r)$. Using (2.5) and (2.10) in (2.8) and (2.9), we get

$$
\phi X=\nabla_{X} \xi_{\alpha}-X+\eta^{\alpha}(X) \xi_{\alpha},
$$

$$
\begin{aligned}
\left(\nabla_{Y} \phi\right)(X)= & -\Sigma_{\alpha} \eta^{\alpha}(X)\left[Y-\eta^{\alpha}(Y) \xi_{\alpha}\right] \\
& -\left[g(X, Y)-\Sigma_{\alpha} \eta^{\alpha}(X) \eta^{\alpha}(Y)\right] \Sigma_{\beta} \xi_{\beta}-g(Y, \phi X) \xi_{\alpha} .
\end{aligned}
$$

\section{Hypersurfaces of almost $r$-paracontact Riemannian manifold endowed with a semi-symmetric metric connection}

Let $M^{n+1}$ be an almost $r$-paracontact Riemannian manifold with a positive definite metric $g$ and $M^{n}$ be a hypersurface immersed in $M^{n+1}$ by the immersion $\tau: M^{n} \rightarrow M^{n+1}$. If $\tau_{*}$ denotes the differential of the immersion $\tau$ and $\bar{X}$ is a vector field on $M^{n}$, then we shall identify $\bar{X}$ and $\tau_{*} \bar{X}$. We denote the objects belonging to $M^{n}$ by the mark of hyphen placed over them, e.g., $\bar{\phi}, \bar{X}, \bar{\eta}, \bar{\xi}$ etc.

Let $N$ be the unit normal vector field to $M^{n}$. The induced metric $\bar{g}$ on $M^{n}$ is defined by

$$
\bar{g}(\bar{X}, \bar{Y})=g(\bar{X}, \bar{Y}) .
$$

Then we have $[6]$

$$
g(\bar{X}, N)=0 \text { and } g(N, N)=1 .
$$

If $\bar{\nabla}^{*}$ be the induced connection on the hypersurface from the Riemannian connection $\nabla^{*}$ in $M^{n+1}$ with respect to the unit normal $N$, then the Gauss and Weingarten formulae are given respectively by

$$
\begin{gathered}
\nabla_{\bar{X}}^{*} \bar{Y}=\bar{\nabla}_{\bar{X}}^{*} \bar{Y}+h(\bar{X}, \bar{Y}) N, \\
\nabla_{\bar{X}}^{*} N=-H(\bar{X}),
\end{gathered}
$$


where $h$ is the second fundamental tensor and $H$ is a tensor field of type $(1,1)$ called the shape operator of $M^{n}$ in $M^{n+1}$ which satisfying

$$
h(\bar{Y}, \bar{X})=h(\bar{X}, \bar{Y})=\bar{g}(H(\bar{X}), \bar{Y}) .
$$

If $\bar{\nabla}$ is the induced connection on the hypersurface from the semi-symmetric metric connection $\nabla$ in $M^{n+1}$ with respect to the unit normal $N$, then we have

$$
\nabla_{\bar{X}} \bar{Y}=\bar{\nabla}_{\bar{X}} \bar{Y}+m(\bar{X}, \bar{Y}) N
$$

where $m$ is a tensor field of type $(0,2)$ of the hypersurface. From $(2.10)$, we obtain

$$
\nabla_{\bar{X}} \bar{Y}=\nabla_{\bar{X}}^{*} \bar{Y}+\eta^{\alpha}(\bar{Y}) \bar{X}-g(\bar{X}, \bar{Y}) \xi_{\alpha}, \quad \alpha \in(r) .
$$

From equations (3.3), (3.4) and (3.5), we get for each $\alpha \in(r)$

$$
\bar{\nabla}_{\bar{X}} \bar{Y}+m(\bar{X}, \bar{Y}) N=\bar{\nabla}_{\bar{X}}^{*} \bar{Y}+h(\bar{X}, \bar{Y}) N+\eta^{\alpha}(\bar{Y}) \bar{X}-g(\bar{X}, \bar{Y})\left(\xi_{\alpha}+a_{\alpha} N\right),
$$

where we put

$$
\xi_{\alpha}=\bar{\xi}_{\alpha}+a_{\alpha} N
$$

By taking the tangential and normal parts respectively from the both sides, we get

$$
\bar{\nabla}_{\bar{X}} \bar{Y}=\bar{\nabla}_{\bar{X}}^{*} \bar{Y}+\eta^{\alpha}(\bar{Y}) \bar{X}-g(\bar{X}, \bar{Y}) \xi_{\alpha}, \quad \alpha \in(r)
$$

and

$$
m(\bar{X}, \bar{Y})=h(\bar{X}, \bar{Y})-a_{\alpha} g(\bar{X}, \bar{Y}) .
$$

Thus we get the following theorem:

Theorem 3.1. The connection induced on a hypersurface of an almost $r$ paracontact Riemannian manifold with semi-symmetric metric connection with respect to the unit normal is also a semi-symmetric metric connection.

From (3.4) and (3.7), we have

$$
\nabla_{\bar{X}} \bar{Y}=\bar{\nabla}_{\bar{X}} \bar{Y}+\left\{h(\bar{X}, \bar{Y})-a_{\alpha} g(\bar{X}, \bar{Y})\right\} N,
$$

which is the Gauss formula for a semi-symmetric metric connection. From equation (3.5), we have

$$
\nabla_{\bar{X}} N=\nabla_{\bar{X}}^{*} N+a_{\alpha} \bar{X}
$$

where

$$
a_{\alpha}=\eta^{\alpha}(N)
$$

From $(3.3)_{2}$ and (3.9), we have

$$
\nabla_{\bar{X}} N=-H \bar{X}+a_{\alpha} \bar{X}
$$

which is the Weingarten formula with respect to semi-symmetric metric connection. 
Now, suppose that $\Sigma=\left(\phi, \xi_{\alpha}, \eta^{\alpha}, \mathrm{g}\right)_{\alpha \in(r)}$ is an almost $r$-paracontact Riemannian structure on $M^{n+1}$. Then every vector field $X$ on $M^{n+1}$ is decomposed as

$$
X=\bar{X}+\lambda(X) N
$$

where $\lambda$ is a 1 -forms on $M^{n+1}$ and $\bar{X}$ is a vector field and $N$ is a normal field on $M^{n}$. Then we have

$$
\begin{gathered}
\phi \bar{X}=\bar{\phi} \bar{X}+b(\bar{X}) N, \\
\phi N=\bar{N}+K N,
\end{gathered}
$$

where $\bar{\phi}$ is a tensor field of type $(1,1), b$ is a 1 -forms and $K$ is a scalar function on the hypersurface $M^{n}$. Now, we define $\bar{\eta}^{\alpha}$ as

$$
\bar{\eta}^{\alpha}(\bar{X})=\eta^{\alpha}(\bar{X}), \quad \alpha \in(r) .
$$

Making use of (3.6), (3.10), (3.12) and (3.13), we obtain from (2.1) through

$$
\begin{gathered}
b(\bar{N})+K^{2}=1-\Sigma_{\alpha}\left(a_{\alpha}\right)^{2}, \\
K a_{\alpha}+b\left(\bar{\xi}_{\alpha}\right)=0, \quad \alpha \in(r), \\
\Phi(\bar{X}, \bar{Y})=\bar{g}(\bar{\phi} \bar{X}, \bar{Y})=\bar{g}(\bar{X}, \bar{\phi} \bar{Y})=\bar{\Phi}(\bar{X}, \bar{Y}) .
\end{gathered}
$$

Making use of (3.1), (3.2), (3.5), (3.12) and (3.13), we have

$$
0=g(\bar{\phi} \bar{X}, N)=g(\phi \bar{X}, N)-b(\bar{X})=g(\bar{X}, \phi N)-b(\bar{X}) .
$$

Hence we get

$$
\bar{g}(\bar{X}, \bar{N})=b(\bar{X}) .
$$

Differentiating covariantly (3.12) and (3.13) along $M^{n}$ and making use of (3.8) and (3.11), we get

$$
\begin{aligned}
\left(\nabla_{\bar{Y}} \phi\right)(\bar{X})= & \left(\bar{\nabla}_{\bar{Y}} \bar{\phi}\right)(\bar{X})-b(\bar{X}) H(\bar{Y})+b(\bar{X}) a_{\alpha} \bar{Y} \\
& -\left(h(\bar{X}, \bar{Y})-a_{\alpha} g(\bar{X}, \bar{Y})\right) \bar{N} \\
+ & +\left[\left(\bar{\nabla}_{\bar{Y}} b\right)(\bar{X})+h(\bar{\phi} \bar{X}, \bar{Y})-K\left(h(\bar{X}, \bar{Y})-a_{\alpha} g(\bar{X}, \bar{Y})\right)\right] N, \\
\left(\nabla_{\bar{Y}} \phi\right) N= & \bar{\nabla}_{\bar{Y}} \bar{N}+\bar{\phi}(H(\bar{Y}))-K H(\bar{Y})-a_{\alpha}(\bar{\phi} \bar{Y}+K \bar{Y}) \\
& +\left[\bar{Y}(K)-2 a_{\alpha} b(\bar{Y})+2 h(\bar{Y}, \bar{N})\right] N .
\end{aligned}
$$

From (3.6) and (3.10), we have

(3.21) $\nabla_{\bar{Y}} \xi_{\alpha}=\bar{\nabla}_{\bar{Y}} \bar{\xi}_{\alpha}-a_{\alpha} H(\bar{Y})+\left(a_{\alpha}\right)^{2} \bar{Y}+\left[\bar{Y}\left(a_{\alpha}\right)+h\left(\bar{Y}, \bar{\xi}_{\alpha}\right)-a_{\alpha} \bar{\eta}^{\alpha}(\bar{Y})\right] N$,

$$
\left(\nabla_{\bar{Y}} \eta^{\alpha}\right)(\bar{X})=\left(\bar{\nabla}_{\bar{Y}} \bar{\eta}^{\alpha}\right)(\bar{X})-a_{\alpha} h(\bar{X}, \bar{Y})+\left(a_{\alpha}\right)^{2} \bar{g}(\bar{X}, \bar{Y}) .
$$


From the identity $\left(\nabla_{Z} \Phi\right)(X, Y)=g\left(\left(\nabla_{Z} \phi\right)(X), Y\right)$, making use of (3.17), (3.18) and (3.19), we have

$$
\begin{aligned}
\left(\nabla_{\bar{Z}} \Phi\right)(\bar{X}, \bar{Y})= & \left(\bar{\nabla}_{\bar{Z}} \bar{\Phi}\right)(\bar{X}, \bar{Y})-b(\bar{X}) h(\bar{Z}, \bar{Y})-b(\bar{Y}) h(\bar{Z}, \bar{X}) \\
& +a_{\alpha} b(\bar{X}) \bar{g}(\bar{Z}, \bar{Y})+a_{\alpha} b(\bar{Y}) \bar{g}(\bar{Z}, \bar{X}) .
\end{aligned}
$$

Theorem 3.2 ([4]). If $M^{n}$ is an invariant hypersurface immersed in an almost $r$-paracontact Riemannian manifold $M^{n+1}$ endowed with semi-symmetric metric connection with structure $\Sigma=\left(\phi, \xi_{\alpha}, \eta^{\alpha}, g\right)_{\alpha \in(r)}$, then either

(i) All $\xi_{\alpha}$ are tangent to $M^{n}$ and $M^{n}$ admits an almost $r$-paracontact Riemannian structure $\Sigma_{1}=\left(\bar{\phi}, \bar{\xi}_{\alpha}, \bar{\eta}^{\alpha}, \bar{g}\right)_{\alpha \in(r)},(n-r>2)$ or

(ii) One of $\xi_{\alpha}\left(\right.$ say, $\left.\xi_{r}\right)$ is normal to $M^{n}$ and remaining $\xi_{\alpha}$ are tangent to $M^{n}$ and $M^{n}$ admits an almost $(r-1)$-paracontact Riemannian structure $\Sigma_{2}=\left(\bar{\phi}, \bar{\xi}_{i}, \bar{\eta}^{i}, \bar{g}\right)_{i \in(r)},(n-r>1)$.

Proof. From (3.15) and (3.16) after computations similar to the computations in the proof of theorem 3.1 in [3] we obtain our theorem.

Corollary 3.1. If $M^{n}$ is a hypersurface immersed in an almost $r$-paracontact Riemannian manifold $M^{n+1}$ with a structure $\Sigma=\left(\phi, \xi_{\alpha}, \eta^{\alpha}, g\right)_{\alpha \in(r)}$ endowed with a semi-symmetric metric connection, then the following statements are equivalent:

(1) $M^{n}$ is invariant.

(2) The normal vector field $N$ is an eigenvector of $\phi$.

(3) All $\xi_{\alpha}$ are tangent to $M^{n}$ if and only if $M^{n}$ admits an almost $r$ paracontact Riemannian structure $\Sigma_{1}$, or one of $\xi_{\alpha}$ is normal and $(r-1)$ remaining $\xi_{i}$ are tangent to $M^{n}$ if and only if $M^{n}$ admits an almost $(r-1)$-paracontact Riemannian structure $\Sigma_{2}$.

Theorem 3.3. If $M^{n}$ is an invariant hypersurface immersed in an almost $r$-paracontact Riemannian manifold of P-Sasakian type endowed with semisymmetric metric connection, then the induced almost $r$-paracontact Riemannian structure $\Sigma_{1}$ or $(r-1)$-paracontact Riemannian structure $\Sigma_{2}$ are also of $P$-Sasakian type.

Proof. Making use of (3.1), (3.14), (3.17), (3.22) and (3.23), we can observe that the conditions (2.11) and (2.12) are satisfied for both $\Sigma_{1}$ and $\Sigma_{2}$.

On the other hand, we have the following.

Lemma 3.1.

$$
\bar{\nabla}_{\bar{X}}(\operatorname{trace} \bar{\phi})=\operatorname{trace}\left(\bar{\nabla}_{\bar{X}} \bar{\phi}\right) \text {. }
$$

Proof. Let $\left\{\mathrm{e}_{1}, \mathrm{e}_{2}, \ldots, \mathrm{e}_{n}\right\}$ be an orthogonal basis of $T M^{n}$ and

$$
\text { trace } \bar{\phi} \stackrel{\text { def }}{=} \Sigma_{a}\left(\bar{\phi}\left(e_{a}\right), e_{a}\right),
$$

where $a \in(n-1)$, then after computations similar to the computations in the proof of Lemma 4.1 in [3] we easily obtain our lemma. 
Theorem 3.4. Let $M^{n}$ be a non-invariant hypersurface of an almost r-paracontact Riemannian manifold $M^{n+1}$ endowed with the semi-symmetric metric connection with a structure $\Sigma=\left(\phi, \xi_{\alpha}, \eta^{\alpha}, g\right)_{\alpha \in(r)}$ satisfying $\nabla \phi=0$ along $M^{n}$. Then $M^{n}$ is totally geodesic if and only if $\left(\bar{\nabla}_{\bar{Y}} \bar{\phi}\right)(\bar{X})+a_{\alpha} \bar{g}(\bar{X}, \bar{Y}) \bar{N}+$ $a_{\alpha} b(\bar{X}) \bar{Y}=0$.

Proof. From (3.19) we have

$$
\begin{gathered}
\left(\bar{\nabla}_{\bar{Y}} \bar{\phi}\right)(\bar{X})-\left(h(\bar{X}, \bar{Y})-a_{\alpha} \bar{g}(\bar{X}, \bar{Y})\right) \bar{N}-b(\bar{X}) H(\bar{Y})+a_{\alpha} b(\bar{X}) \bar{Y}=0 \\
\left(\bar{\nabla}_{\bar{Y}} b\right)(\bar{X})+a_{\alpha} \bar{g}(\bar{X}, \bar{Y})+h(\bar{Y}, \bar{\phi} \bar{X})-K h(\bar{X}, \bar{Y})=0
\end{gathered}
$$

If $M^{n}$ is totally geodesic, then $h=0$ and $H=0$, so we get from (3.24),

$$
\left(\bar{\nabla}_{\bar{Y}} \bar{\phi}\right)(\bar{X})+a_{\alpha} \bar{g}(\bar{X}, \bar{Y}) \bar{N}+a_{\alpha} b(\bar{X}) \bar{Y}=0 .
$$

Conversely, if $\left(\bar{\nabla}_{\bar{Y}} \bar{\phi}\right)(\bar{X})+a_{\alpha} \bar{g}(\bar{X}, \bar{Y}) \bar{N}+a_{\alpha} b(\bar{X}) \bar{Y}=0$, then

$$
h(\bar{X}, \bar{Y}) \bar{N}+b(\bar{X}) H(\bar{Y})=0 .
$$

Making use of (3.18), we have

$$
b(\bar{Z}) h(\bar{X}, \bar{Y})+b(\bar{X}) h(\bar{Y}, \bar{Z})=0 .
$$

Using (3.25), we get

$$
b(\bar{X}) h(\bar{Y}, \bar{Z})=b(\bar{Y}) h(\bar{X}, \bar{Z}) .
$$

From (3.26) and (3.27), we get $b(\bar{Z}) h(\bar{X}, \bar{Y})=0$ which gives that $h=0$ as $b \neq 0$. Using $h=0$ in (3.25), we get $H=0$. Thus, $h=0$ and $H=0$. Hence $M^{n}$ is totally geodesic.

Also we have the following:

Theorem 3.5. Let $M^{n}$ be a non-invariant hypersurface of an almost r-paracontact Riemannian manifold $M^{n+1}$ with semi-symmetric metric connection satisfying $\nabla \phi=0$ along $M^{n}$ and trace $\bar{\phi}=$ constant, then $M^{n}$ is totally umbilical.

Proof. From (3.24) we have

$$
\bar{g}\left(\left(\bar{\nabla}_{\bar{Y}} \bar{\phi}\right)(\bar{X}), \bar{X}\right)=2 h(\bar{X}, \bar{Y}) b(\bar{X})-2 a_{\alpha}(\bar{X}) g(\bar{X}, \bar{Y})
$$

and

$$
\bar{\nabla}_{\bar{X}}(\operatorname{trace} \bar{\phi})=\Sigma_{a} \bar{g}\left(\bar{\nabla} \overline{\bar{X}} \bar{\phi}\left(e_{a}\right), e_{a}\right) .
$$

Using Lemma 3.1, we get

$$
h(\bar{X}, \bar{N})=a_{\alpha} \Sigma_{a} b\left(e_{a}\right) \bar{g}\left(\bar{X}, e_{a}\right),
$$

where $\bar{N}=\Sigma_{a} b\left(e_{a}\right) e_{a}$, which implies that $M^{n}$ is totally umbilical. 
Now, let $M^{n+1}$ be an almost $r$-paracontact Riemannian manifold of $S$ paracontact type. Then from (2.8), (3.12) and (3.21), we get

$$
\begin{gathered}
\bar{\phi} \bar{X}=\bar{\nabla}_{\bar{X}} \bar{\xi}_{\alpha}-a_{\alpha} H(\bar{X})+\left(a_{\alpha}\right)^{2}(\bar{X})-\bar{X}+\bar{\eta}^{\alpha}(\bar{X}) \bar{\xi}_{\alpha}, \quad \alpha \in(r), \\
b(\bar{X})=\bar{X}\left(a_{\alpha}\right)+h\left(\bar{X}, \bar{\xi}_{\alpha}\right), \quad \alpha \in(r) .
\end{gathered}
$$

Making use of (3.29), we have that if $M^{n}$ is totally geodesic, then $a_{\alpha}=0$ and $h=0$. Hence $b=0$, that is, $M^{n}$ is invariant. Thus we have:

Proposition 3.1. If $M^{n}$ is totally geodesic hypersurface of an almost r-paracontact Riemannian manifold $M^{n+1}$ endowed with the semi-symmetric metric connection of $S$-paracontact type with a structure $\Sigma=\left(\phi, \xi_{\alpha}, \eta^{\alpha}, g\right)_{\alpha \in(r)}$ and all $\xi_{\alpha}$ are tangent to $M^{n}$, then $M^{n}$ is invariant.

Theorem 3.6. If $M^{n}$ is an anti-invariant hypersurface of an almost r-paracontact Riemannian manifold $M^{n+1}$ endowed with the semi-symmetric metric connection of $S$-paracontact type with a structure $\Sigma=\left(\phi, \xi_{\alpha}, \eta^{\alpha}, g\right)_{\alpha \in(r)}$, then $\bar{\nabla}_{\bar{X}} \xi_{\alpha}=\phi^{2} \bar{X}$.

Proof. If $M^{n}$ is anti-invariant, then $\bar{\phi}=0$ and $a_{\alpha}=0$ and also from (3.28) we have

$$
\bar{\nabla}_{\bar{X}} \bar{\xi}_{\alpha}=\bar{X}-\bar{\eta}^{\alpha}(\bar{X}) \bar{\xi}_{\alpha}, \quad \alpha \in(r)
$$

That is,

$$
\bar{\nabla}_{\bar{X}} \xi_{\alpha}=\phi^{2} \bar{X}
$$

Now, let $M^{n+1}$ be an almost $r$-paracontact Riemannian manifold endowed with the semi-symmetric metric connection of P-Sasakian type. Then from (2.11) and (3.19), we have

$$
\begin{aligned}
&\left(\bar{\nabla}_{\bar{Y}} \bar{\phi}\right)(\bar{X})-\left[h(\bar{X}, \bar{Y})-a_{\alpha} \bar{g}(\bar{X}, \bar{Y})\right] \bar{N}-b(\bar{X}) H(\bar{Y})+a_{\alpha} b(\bar{X}) \bar{Y} \\
&(3.30)=-\Sigma_{\alpha} \bar{\eta}^{\alpha}(\bar{X})\left[\bar{Y}-\bar{\eta}^{\alpha}(\bar{Y}) \bar{\xi}_{\alpha}\right]-\left[\bar{g}(\bar{X}, \bar{Y})-\Sigma_{\alpha} \bar{\eta}^{\alpha}(\bar{X}) \bar{\eta}^{\alpha}(\bar{Y})\right] \Sigma_{\beta} \bar{\xi}_{\beta} .
\end{aligned}
$$

Theorem 3.7. Let $M^{n+1}$ be an almost $r$-paracontact Riemannian manifold of P-Sasakian type with a structure $\Sigma=\left(\phi, \xi_{\alpha}, \eta^{\alpha}, g\right)_{\alpha \in(r)}$ endowed with the semi-symmetric metric connection and let $M^{n}$ be a hypersurface immersed in $M^{n+1}$ such that none of $\xi_{\alpha}$ is tangent to $M^{n}$. Then $M^{n}$ is totally geodesic if and only if

$\left(\bar{\nabla}_{\bar{Y}} \bar{\phi}\right)(\bar{X})+a_{\alpha} b(\bar{X}) \bar{Y}-a_{\alpha} \bar{g}(\bar{X}, \bar{Y}) \bar{N}$
$(3.31)=-\Sigma_{\alpha} \bar{\eta}^{\alpha}(\bar{X})\left[\bar{Y}-\bar{\eta}^{\alpha}(\bar{Y}) \bar{\xi}_{\alpha}\right]-\left[\bar{g}(\bar{X}, \bar{Y})-\Sigma_{\alpha} \bar{\eta}^{\alpha}(\bar{X}) \bar{\eta}^{\alpha}(\bar{Y})\right] \Sigma_{\beta} \bar{\xi}_{\beta}$.

Proof. If (3.31) is satisfied, then from (3.30), we get $h(\bar{X}, \bar{Y}) \bar{N}+b(\bar{X}) H(\bar{Y})=0$. Since $b \neq 0$ so that by use of Theorem $3.4, h(\bar{X}, \bar{Y})=0$. Hence $M^{n}$ is totally geodesic. Conversely, Let $M^{n}$ is totally geodesic, that is $h(\bar{X}, \bar{Y})=0, H=0$, then from (3.29) we have $b=0$, which is a contradiction. Hence $\xi_{\alpha}$ are not tangent to $M^{n}$. 
Acknowledgement. The authors are grateful to the referee for his/her kind suggestions.

\section{References}

[1] M. Ahmad, J.-B. Jun, and A. Haseeb, Hypersurfaces of almost $r$-paracontact Riemannian manifold endowed with a quarter symmetric metric connection, Bull. Korean Math. Soc. 46 (2009), no. 3, 477-487.

[2] M. Ahmad and C. Ozgur, Hypersurfaces of almost r-paracontact Riemannian manifold endowed with a semi-symmetric non-metric connection, Results in Mathematics, Accepted.

[3] A. Bucki, Hypersurfaces of almost r-paracontact Riemannian manifolds, Tensor (N.S.) 48 (1989), no. 3, 245-251.

[4] _ Almost r-paracontact structures of P-Sasakian type, Tensor (N.S.) 42 (1985), no. $1,42-54$.

[5] A. Bucki and A. Miernowski, Almost r-paracontact structures, Ann. Univ. Mariae CurieSklodowska Sect. A 39 (1985), 13-26.

[6] B. Y. Chen, Geometry of Submaifolds, Marcel Dekker, New York, 1973.

[7] A. Friedmann and J. A. Schouten, Über die geometrie der halbsymmetrischen ubertrangung, Math. Z. 21 (1924), no. 1, 211-223.

[8] J. A. Schouten, Ricci Calculus, Springer, 1954.

[9] K. Yano, On semi-symmetric metric connection, Rev. Roumaine Math. Pures Appl. 15 (1970), 1579-1586.

JAE-BOK JUN

Department of Mathematics

College of Natural Science

KOOK-Min University

SEOUl 136-702, Korea

E-mail address: jbjun@kookmin.ac.kr

Mobin Ahmad

Department of Mathematics

INTEGRAL UNIVERSITY

KuRSi-RoAd, LuCKNOW-226026, IndiA

E-mail address: mobinahmad@rediffmail.com 